\title{
Particle Filter for Hector SLAM to Improve the Performance of Robot Positioning by Image Processing Based
}

\author{
W. Sirigool and R. Kesvarakul
}

\begin{abstract}
Today, robot navigation uses navigation from maps that have been collected from laser sensors. The quality and usability of the received environmental grid map. This has an evident impact on robot navigation. The more accurate the map is a direct effect on the autonomous navigation. The morphological operation was used to an improved map by using opening and closing operations to reduce noise from the original map. This paper presents an image processing technique. In order to, robots be able to reduce the time required for the navigation to the destination due to inaccuracy maps.
\end{abstract}

Index Terms-Morphological, opening, closing, Hector SLAM.

\section{INTRODUCTION}

Nowadays, robots have become increasingly important in human life. Autonomous robots are used widely in many industries. Localization and navigation are the core technologies of autonomous mobile robots. In this period, a low-cost laser rangefinder is widely used in autonomous mobile robots due to a very cheap price.

The navigation process for autonomous mobile robots can be divided into four steps: environment mapping, motion control, trajectory design, and obstacle avoidance. Robot navigation in a dynamic environment can be divided into two main categorize which is create more accuracy map and generate safe path navigation and obstacle avoidance. The Simultaneous Localization and Mapping (SLAM) algorithm can be used to build the environment map while are moving and positioning themselves at the same time. Slam algorithm can be divided into two major categories; one is Filter-based approaches, examples of filter-based algorithms are: Fast SLAM, Hector SLAM, EKF-SLAM, UKF SLAM, Etc.; the other is optimization-based SLAM, examples of filter-based algorithms are: Graph-based SLAM [1]-[4], TreeMap, TORO. Hector SLAM was selected for this paper because this algorithm is use only laser rangefinder with high frequency scanning rates is necessary.

Hector SLAM [5], it is based on the comparison of the scanned data, while scan matching the data will be already created map. The Gauss-Newton Algorithm solved data comparison data form laser rangefinder. It attempts to find position of laser scan endpoints with created map. The great

Manuscript received January 10, 2020; revised March 2, 2020

The authors are with Production Engineering Department, King Mongkut's University of Technology North Bangkok, 1518 Pracharat 1 Road, Wongsawang, Bangsue, Bangkok 10800 Thailand (e-mail: watcharapong_s@hotmail.com,ramil.k@eng.kmutnb.ac.th). advantage of this method is that robot odometry is not necessary. On the other hand, the disadvantage of Hector SLAM is that in the case of a combination of the slow frequency rangefinder rates, the lack of odometry and the rapid movement of robot, causes the map to be inaccurate. The hector SLAM with a hybrid (PF/FIR) algorithm for robot positioning environments. The result showed the hybrid filter provides a more accurate, continuous, and reliable position approximation [6]. Reasons to make the map update to be accurate. So the robot can specify the correct position on the map and efficiently navigate the robot. Therefore, the idea of using image processing to improve a map precisely by reduce noise problems that appear on the map during scanning of environmental data from the laser rangefinder.

In this paper, we propose morphological operation to improve the map image for autonomous robot navigation. The opening and closing are achieved to improve map image. The paper is structured as follows: Section II presents the morphological operations. Section III presents the hector SLAM. The experiment procedure and results are described in Section IV and Section V sequentially. The conclusion is the final section.

\section{HECTOR SLAM}

Hector SLAM algorithm is selected as the structure for this work. This SLAM algorithm does not require odometer for create map and localization. The coordinate setting with the $\mathrm{z}$-axis pointing upwards and the $\mathrm{x}$-axis pointing into the forward direction of a robot was used a long-range laser rangefinder for this method.

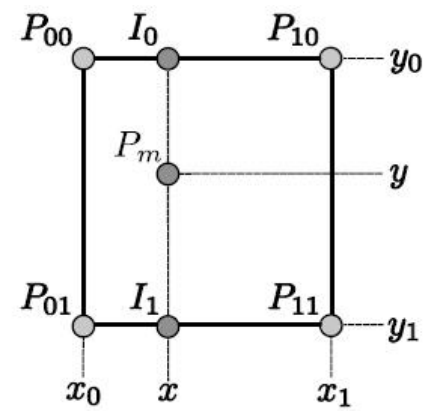

Fig. 1. Bilinear filtering of the occupancy grid map. $P_{m}$ is a map coordinate.

The map available is the occupancy grid maps, which has a discrete nature limitation of the precision that is not directly accessible and will be achieved by computation of interpolated values or derivatives. Bilinear filtering is employed for interpolating sub-grid cell value to estimating 
occupancy probabilities and derivatives. In this way, the grid map separate value surface is continuous in any single point of the map as shown in Fig. 1.

Given a point in a continuous map, $P_{m}$, the occupancy value, $\left(P_{m}\right)$, the gradient will be in the form:

$$
\nabla M\left(P_{m}\right)=\left(\left(\frac{\partial M}{\partial x}\right)\left(P_{m}\right),\left(\frac{\partial M}{\partial y}\right)\left(P_{m}\right)\right)
$$

The occupancy value is approximated by using the bilinear method, the linear interpolation with closest integer coordinates $P_{00}, P_{10}, P_{10}, 11$ can be defined as

$$
\begin{aligned}
M\left(P_{m}\right) & \approx \frac{y-y_{0}}{y_{1}-y_{0}}\left(\frac{x-x_{0}}{x_{1}-x_{0}} M\left(P_{11}\right)+\frac{x-x_{0}}{x_{1}-x_{0}} M\left(P_{01}\right)\right) \\
& +\frac{y-y_{0}}{y_{1}-y_{0}}\left(\frac{x-x_{0}}{x_{1}-x_{0}} M\left(P_{10}\right)+\frac{x-x_{0}}{x_{1}-x_{0}} M\left(P_{00}\right)\right)
\end{aligned}
$$

The derivatives can be approximated by:

$$
\begin{gathered}
\left(\frac{\partial M}{\partial x}\right)\left(P_{m}\right) \approx \frac{y-y_{0}}{y_{1}-y_{0}}\left(M\left(P_{11}\right)-M\left(P_{01}\right)\right) \\
+\frac{y_{1}-y}{y_{1}-y_{0}}\left(M\left(P_{10}\right)-M\left(P_{00}\right)\right) \\
\left(\frac{\partial M}{\partial y}\right)\left(P_{m}\right) \approx \frac{x-x_{0}}{x_{1}-x_{0}}\left(M\left(P_{11}\right)-M\left(P_{10}\right)\right) \\
+\frac{x_{1}-x}{x_{1}-x_{0}}\left(M\left(P_{01}\right)-M\left(P_{00}\right)\right)
\end{gathered}
$$

Scanning matching is a process for sorting a laser scan. Each other or with an existing map Modern laser scanner There is noise, low distance measurement and high scan rate. Scan matching is based on optimization of the alignment of beam endpoints with the map obtained. The basic idea using a Gauss-Newton approach is utilized to predict the next pose without search data between the endpoint. The rigid transformation $\xi=\left(P_{x}, P_{y}, \psi\right)$ that minimizes:

$$
\xi^{*}=\underset{\xi}{\operatorname{argmin}} \sum_{i=1}^{n}\left[1-M\left(S_{i}(\xi)\right)\right]^{2}
$$

where is $S_{i}(\xi)$ are the world coordinates of scan endpoint. $s_{i}$ $=\left(s_{i ; x} ; s_{i ; y}\right) T$ They are a function of $\xi$, the pose of the robot in world coordinates.

$$
S_{i}(\xi)\left(\begin{array}{cr}
\cos (\psi)-\sin (\psi) \\
\sin (\psi) & \cos (\psi)
\end{array}\right)\left(\begin{array}{c}
S_{i, x} \\
S_{i, y}
\end{array}\right)+\left(\begin{array}{c}
P_{x} \\
P_{y}
\end{array}\right)
$$

The function $M\left(S_{i}(\xi)\right)$ returns the map value at the coordinates given by $S_{i}(\xi)$ given some starting estimate of $\xi$, we want to estimate $\Delta \xi$ which optimizes the error measure according to

$$
\sum_{i=1}^{n}\left[1-M\left(S_{i}(\xi+\Delta \xi)\right)\right]^{2} \rightarrow 0 .
$$

Hence, applying first order Taylor expansion to $M\left(S_{i}\right.$ $(\xi+\Delta \xi))$ i M S and setting the partial derivative with respect to $\Delta \xi$ to zero leads to the minimize value in (8)

$$
\Delta \xi=H^{-1} \sum_{i=1}^{n}\left[\nabla M\left(S_{i}(\xi)\right) \frac{\partial S_{i}(\xi)}{\partial \xi}\right]^{T}\left[1-M\left(S_{i}(\xi)\right)\right]
$$

where

$$
H=\left[\nabla M\left(S_{i}(\xi)\right) \frac{\partial S_{i}(\xi)}{\partial \xi}\right]^{T}\left[\nabla M\left(S_{i}(\xi)\right) \frac{\partial S_{i}(\xi)}{\partial \xi}\right]
$$

\section{MORPHOLOGICAL OPERATIONS}

Mathematical morphological operations are commonly used as a tool in image processing [7]. The researcher enhanced the document images using various morphological techniques that use a part of morphological erosion, dilation, closing, and opening. The document image was improved by using various morphological techniques 2 various types of handwriting images for this analysis. In [8] present a method detect edge from depth image by using a depth image smoothing algorithm and Canny edge detection algorithm. The results show that the level of noise was minimized by using the morphological operation that is median filtering principle was applied to a smooth algorithm. Moreover, while eliminating additional noise, the contour lines are smooth and the object's shape and size are maintained. The shape of various objects in the scene is preserved. In [9] presents an image processing technique, which is a morphological operation. The 4 morphological operations (dilation, erosion, opening, and closing) and 2 basic algorithms, that are boundary extraction and region filling, are applied in Matlab program. The 3 types of stricter element, that are square, diamond and octagon, were used to investigate the effect of image restoration base on morphological. The simple technique of morphological operations can improve binary image and reduce noise [10], ]12].

\section{A. Dilation}

The dilation is a basic operation in morphological operation. This operation will increase the size of the pixels around all features and regions, which will cause a slight increase in dimensions and may cause the merged pixel. It will also fill a small hole inside the feature. The dilation of an image A (set) by structuring element $\mathrm{B}$ is defined as

$$
\left.A \oplus B=\{z \widehat{(B)})_{z} \cap A \neq \emptyset\right\}
$$

In Fig. 2, the dilation process is basically used to fill the holes in a continuous object. The dilation operation, as it increases the pixel at the boundary of the object, it affects the intensity at that location and can observe the blur effect of the image. The dilation process expands the number of pixels with one value (white) and reduces the number of pixels to zero (black).

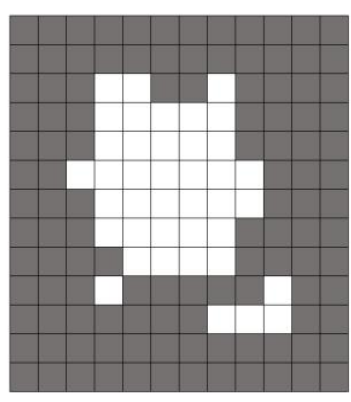

(a)

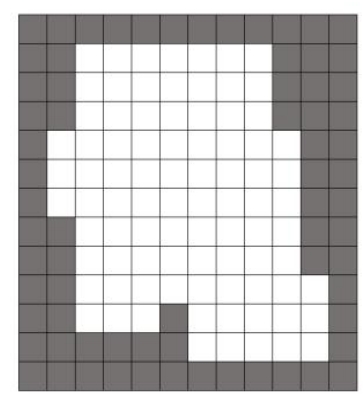

(b)
Fig. 2. Dilation operation: (a). Original image (b). Dilation image.

\section{B. Erosion Can Entirely Remove Extraneous Pixels Representing Erosion}

point noise that is the opposite operation of dilation. The erosion of an image A by structuring element $\mathrm{B}$ is defined as 


$$
A \ominus B=\left\{z(B)_{z} \subseteq A\right\}
$$

The erosion of image $A$, where the structural element $B$ is the set of all points $z$, in which the structural element $B$ is interpreted as $\mathrm{z}$ as a subset of the image. This action results in a loss of pixel boundaries of the object. The erosion process expands the number of pixels to zero (background) and reduces the number of pixels by one value (foreground). In Fig. 3, the erosion operation removes those structures which are smaller in size than that of the structuring element.

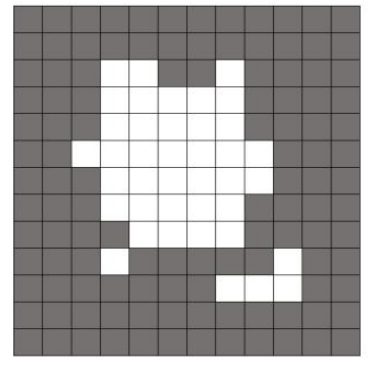

(b)

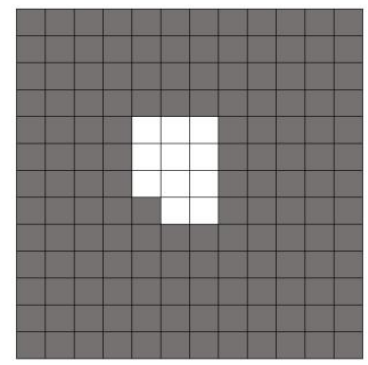

(a)

Fig. 3. Erosion operation: (a). Original image (b). Erosion image.

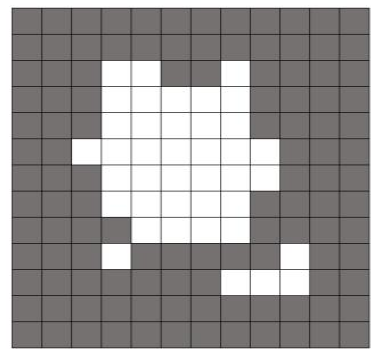

(a)

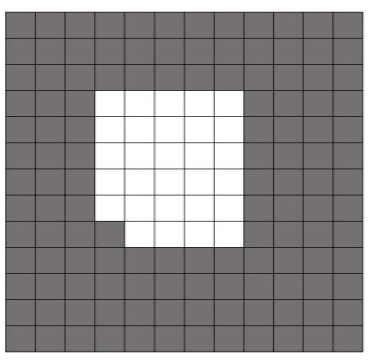

(b)
Fig. 4. Opening operation: (a). Original image (b). Opening image.

\section{Opening}

The opening of an image is a combinational operation of erosion and dilation. The opening of an image A by structuring element $\mathrm{B}$ is defined as

$$
A \circ B=(A \ominus B) \oplus B
$$

The Opening operations involve erosion followed by dilation, while closing operations begin with dilation followed by erosion. The opening operation smooths the outline of an object clears the narrow bridges and also eliminates minor extensions present in the object as show in Fig. 4.

\section{Closing}

As shown in Fig. 5, closing images is also a combinational operation of erosion and dilation. It is different from the open operation in the sense of the order of occurrence of erosion and dilation. The closing of an image A by structuring element $\mathrm{B}$ is defined as

$$
A \cdot B=(A \oplus B) \ominus B
$$

The relation between erosion $\&$ dilation with closing is given in the above mathematical statement. It shows that closing operation is the dilation of an image A by the structuring element B and the resultant is eroded with the same structuring element. Close operations smooth out various parts of the shape. In general, it will divide the spaces narrow and thinner, resulting in it eliminating small holes and filling gaps in the object of a boundary.

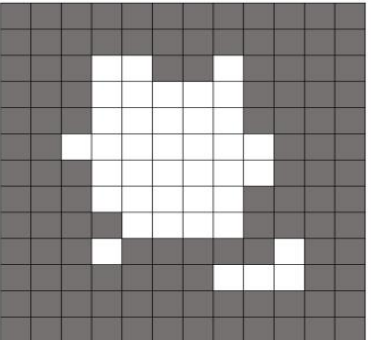

(a)

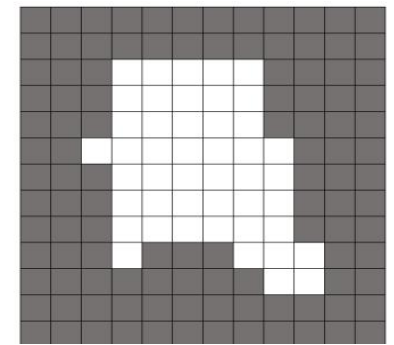

(b)
Fig. 5. Closing operation: (a). Original image (b). Closing image.

\section{EXPERIMENT PROCEDURE}

The experiment procedure was divide three part, which is prepare setup step, image processing step and verification step, as show in Fig. 6.

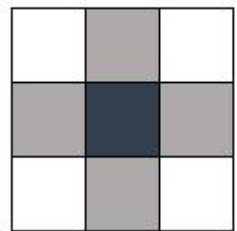

(a)

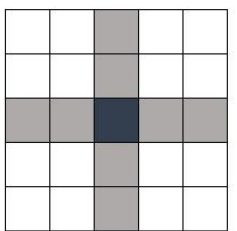

(b)

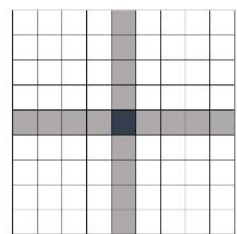

(c)
Fig. 6. Structuring element size of morphological at different element (a) 3element (b) 5 element (c) 9 element.

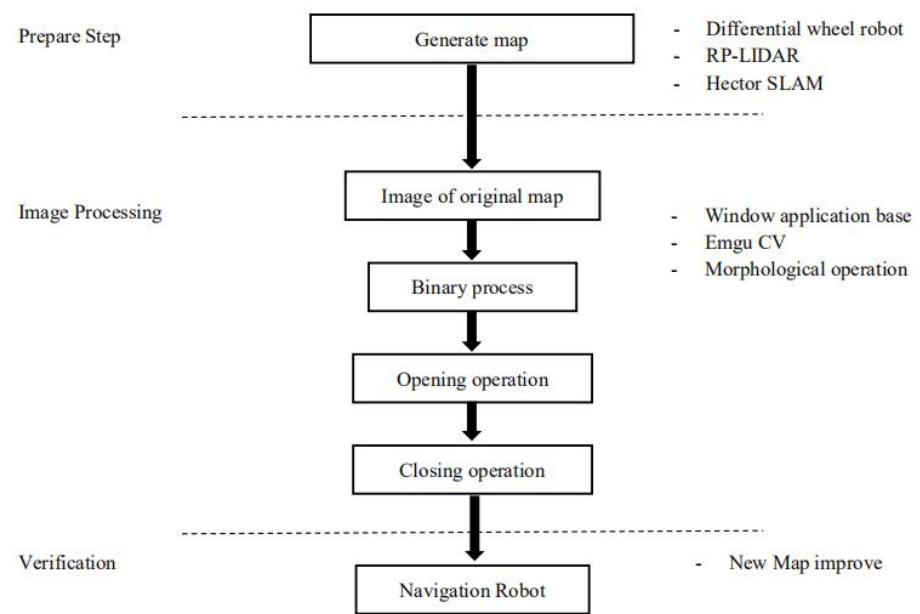

Fig. 7. Overall of the experimental procedure. 

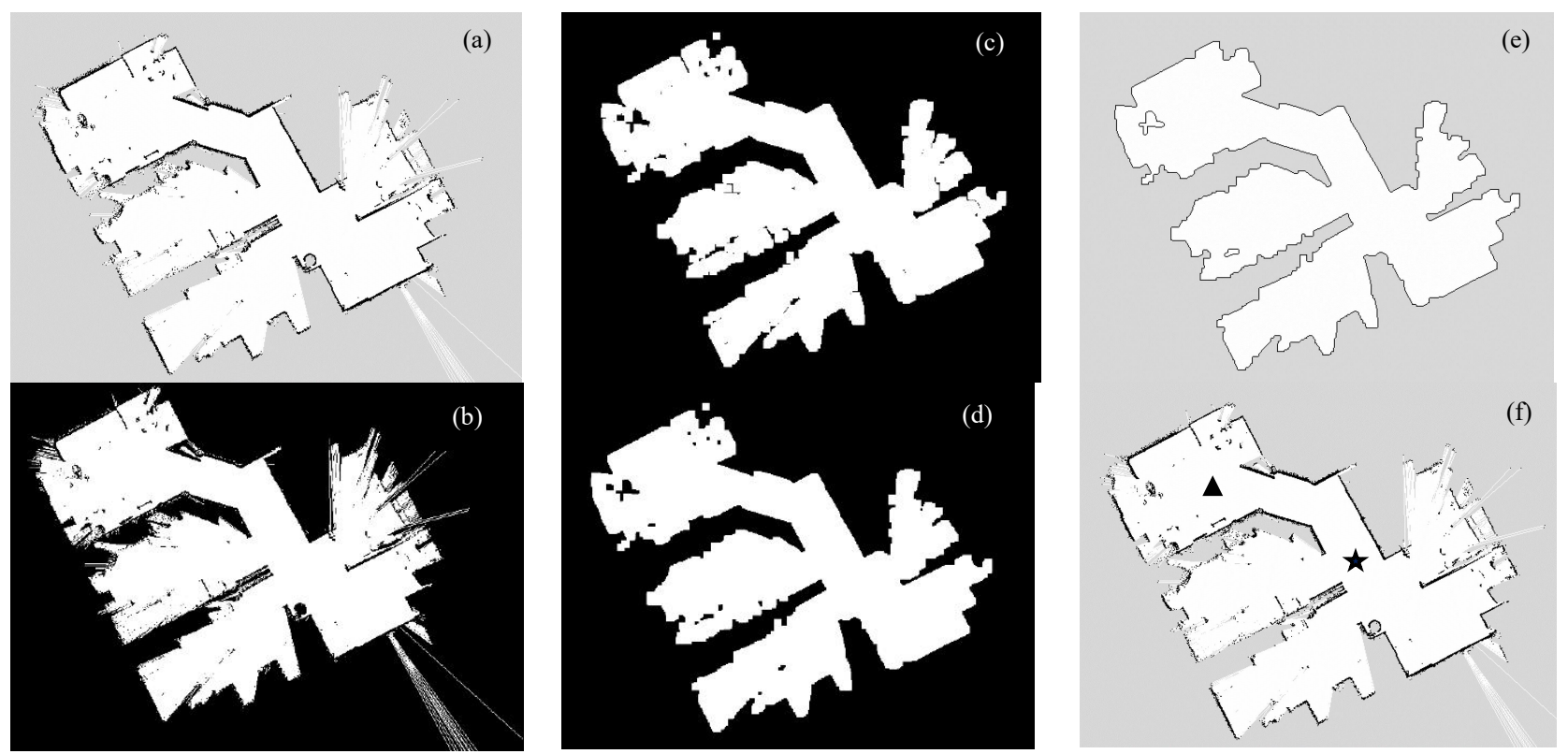

Fig. 8 The map images at each step have been improved by a morphological operation. (a) Original map, (b) Binary map, (c) Closing map, (d) Opening map, (e) Final map, (f) The map show a start point in black triangle and goal point in black star for robot navigation.

\section{A. Prepare Step}

The mobile robot used in our experiments is the differential two wheel robot. The robot can be applied RP-LiDAR can detect the distance of the obstacles with a 360 degree view. The computational platform has Intel Atom processor and $2 \mathrm{G}$ memory, running on Ubuntu $16+$ ROS Kinetic system. The robot operating system uses control a robot, which is used to send and receive, each database will have sensor data received by the robot while running in the actual location, including laser scan data. Hector SLAM algorithm was used create a map by using RP-LIDAR.

\section{B. Image Processing}

We developed the image process program especially for this research by visual studio program. Also ,the EmguCV is a cross-platform .Net wrapper to the OpenCV image processing library. Allowing OpenCV functions to be called from .NET compatible language that is visual basic. The principle of program brings an original map from Hector SLAM as shown in Fig. 8(a) change to a binary image. Thresholding is the simplest method of segmenting image. From an original image, thresholding used to create binary images as shown in Fig. 8(b). the threshold processing of the map image has reduced the level of noise using an image processing technique.

Both opening and closing operations were applied structuring elements of size $3 \times 3,5 \times 5$ and $9 \times 9$ over the image as shown in Fig. 7. The opening operation clears the narrow bridges and also eliminates minor extensions present in the image as shown in Fig. 8(c). Later, the closing operation eliminating small holes and filling gaps in the object of a boundary as shown in Fig. 8(d). The final map goes through a morphological process that creates boundaries. The new map is different from the original map as shown in Fig. 8(e).

\section{Verification}

The new map was used for navigation robot. All 9 maps are used to determine the efficiency of the morphological operation by time consumption of robot moving from the beginning to goal position as shown in Fig. 8(f). Each map was used to investigate the proper parameter of structure element size verify by time consumption when a robot was navigation from a starting point to goal point.

\section{RESULT}

This paper experiments on four different morphological operations: dilation, erosion, opening, and closing operations. Morphological operations in this paper are used for noise removal and to produce the best possible representation of the new map of robot navigation. The three sizes of an element that reduce noise from the original image.

To validate the presented system and to identify the morphological operation performance. The goal is to improve the map using a morphological operation. Also, a map improvement uses to estimate the navigation system needs to be improved upon as shown in Table I.

TABLE I: The Results of EXECUTION TIME FOR RoBot NAVIGATION BY MORPHOLOGICAL OPERATIONS

\begin{tabular}{|c|l|l|}
\hline Image Number & Opening and Closing Operation & Time(sec.) \\
\hline 1 & Original Map & 80 \\
\hline 2 & Opening $3 \times 3$ Closing $3 \times 3$ & 58 \\
\hline 3 & Opening $3 \times 3$ Closing $5 \times 5$ & 65 \\
\hline 4 & Opening $3 \times 3$ Closing $9 \times 9$ & 75 \\
\hline 5 & Opening $5 \times 5$ Closing $3 \times 3$ & 62 \\
\hline 6 & Opening $5 \times 5$ Closing $5 \times 5$ & 70 \\
\hline 7 & Opening $5 \times 5$ Closing $9 \times 9$ & 73 \\
\hline 8 & Opening $9 \times 9$ Closing $3 \times 3$ & 64 \\
\hline 9 & Opening $9 \times 9$ Closing $5 \times 5$ & 66 \\
\hline 10 & Opening $9 \times 9$ Closing $9 \times 9$ & 65 \\
\hline
\end{tabular}

Table I shows that the execution time for robot navigation 
can reduce the time by 22 seconds when using the map number 2 by morphological operations. In addition, the second map the execution time also decreased.

Morphological operations are useful in many applications. A hole was filled, boundary extraction of objects, extraction of connected components. Among these applications, the boundary extraction is shown in Fig. 9. The original map and improvement maps, which used morphological technique, were compared. It can be seen that noise and lines have been clearly eliminated. The details of the map will decrease as the SE increases. Therefore, the results of using SE at $9 \times 9$ will cause the map to be very different from the original map.

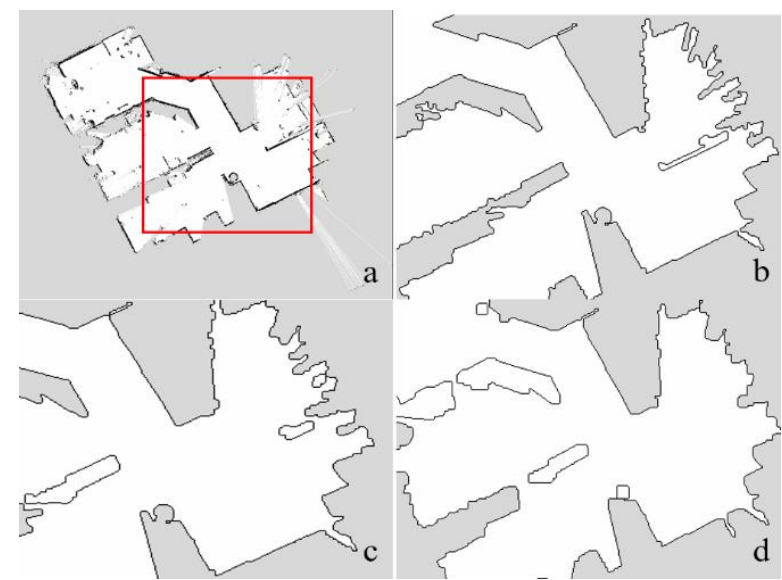

Fig. 9 Map improved by opening and closing operation. (a) Original image (b) Opening $3 \times 3$ Closing $3 \times 3$ (c) Opening $3 \times 3$ Closing $5 \times 5$ (d) Opening 3 $\times 3$ Closing $9 \times 9$.

\section{CONCLUSION}

In this paper, the attempt has been made to understand the basics of the morphological operations used for image processing. The morphological operation erosion and dilation in open and close operations, there are many benefits in reducing noise. They can add and delete pixels.

The experiment result of the execution time of robot navigation shows, the enhancement image affects movement time, the proper parameters of morphological operations. The $3 \times 3$ kernel (opening $3 \times 3$, closing $3 \times 3$ ) is suitable to be used to improve the map image because the map image has not been lost or deviated much from the original image, so its use in robot navigation is highly effective. As shown in Fig. 8, it can be seen that the map is very different from the original image, so there is a chance that navigation problems will not be able to dodge obstacles because the obstacles have been removed from the map.

\section{CONFLICT OF INTEREST}

The authors declare no conflict of interest.

\section{AUTHOR CONTRIBUTIONS}

The first author is tasked with collecting data, analyzing data, and analyzing results until conclusions are obtained. While the second author guides and provides suggestions for each stage of research. All authors had approved the final

version.

\section{REFERENCES}

[1] P. Oksa, P. Loula, and E. Castrén, "Mapping, localization and navigation improvements by using manipulated floor plan and ROS-based mobile robot parameters," Multidisciplinary Engineering Science and Technology, vol. 5, issue 10, pp. 8910-8918, October 2018.

[2] S. Khan, D. Wollherr, and M. Buss, "Modeling laser intensities for simultaneous," IEEE Robotics and Automation Letters, vol. 1, no. 2, pp. 692-699, July 2016

[3] H. Peel, S. Luo, A. G. Cohn, and R. Fuentes, "An improved robot for bridge inspection," in Proc International Symposium on Automation and Robotics in Construction, 2017.

[4] G. Grisetti et al., "A tutorial on graph-based SLAM," IEEE Intelligent Transportation Systems, vol. 2, no. 4, pp. 31-43, December 2010

[5] Y. Abdelrasoul, A. B. S. H. Saman, and P. Sebastian," A quantitative study of tuning ROS gmapping parameters and their effect on performing indoor 2D SLAM," in Proc International Symposium on Robotics and Manufacturing Automation (ROMA), September 2016, pp. 1-6.

[6] A. Bassiri, M. A. Oskoei, and A. Basiri, "Particle filter and finite impulse response filter fusion and hector SLAM to improve the performance of robot positioning," Hindawi Journal of Robotics, pp. 1-9, November 2018.

[7] A. Sakila and S. Vijayarani, "Image enhancement using morphological operations," IJSRSET, vol. 3, pp. 685-698, April 2017.

[8] S. M. A. Hasan and K. Ko, "Depth edge detection by image-based smoothing and morphological operations," Computational Design and Engineering, pp. 191-197, February 2016.

[9] A. M. Raid, W. M. Khedr, M. A. El-dosuky and Mona Aoud, "Image restoration based on morphological operations," Computer Science, Engineering and Information Technology, vol. 4,no. 3, pp. 9-21, June 2014.

[10] E. R. Davies, Computer and Machine Vision: Theory, Algorithms, Practicalities, Oxford: Elsevier, 2002, ch. 7.

[11] N. Jamil, T. M. Tengku Sembok, and Z. A. Bakar, "Noise removal and enhancement of binary images using morphological operations," in Proc. International Symposium on Information Technology, 2008.

[12] S. Ravi and A. M. Khan, "Morphological operations for image processing," in Proc. NCVSComs, 2013, pp. 17-19.

Copyright $(\odot 2020$ by the authors. This is an open access article distributed under the Creative Commons Attribution License which permits unrestricted use, distribution, and reproduction in any medium, provided the original work is properly cited (CC BY 4.0).

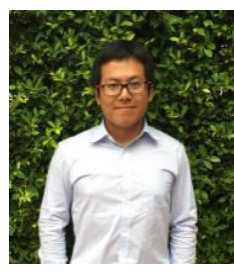

processing.

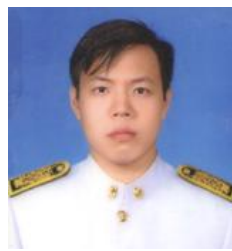

Ramil Kesvarakul received the M.Eng degree in automotive engineering from King Mongkut's Institute of Technology Ladkrabang, Bangkok, Thailand, in 2010 and the Ph.D. degree in industrial engineering from King Mongkut's University of Technology North Bangkok, Bangkok, Thailand, in 2014, respectively. His research interests include the automation system, finite element analysis, image processing, metal forming process, computer programming (basic, $\mathrm{C}, \mathrm{C \#}$, python), tubehydro forming, computer aided design, computer aided engineering, computer aided manufacturing. He is currently an assistance professor at The Department of Production Engineering of at King Mongkut's University of Technology North Bangkok. 